\title{
Properties and Preparation of High Entropy Alloys
}

\author{
Xiang Yin ${ }^{1}$ and Shuqiong $\mathrm{Xu}^{1 *}$ \\ ${ }^{1}$ School of Mechanical and Vehicle Engineering, Linyi University, Shandong, China
}

\begin{abstract}
As a consequence of multi-components, the high entropy alloys embodied serious cocktail party effect and lattice distortion. So high entropy alloys have high strength and hardness possess many comprehensive properties such as thermostability and corrosion resistance. Because of excellent mechanical properties and chemical properties, high entropy alloys have immeasurable potential of development. This paper mainly introduces the properties, preparations and applications of high entropy alloys, and finally summarizes them.
\end{abstract}

\section{Introduction}

From the traditional concepts' perspective, excessive components will give rise to degradation of integrated performance. After the proposal of Yeh. J .W [1] in 2004 there are so many excellent researchers carrying out extensive researches and explorations. A lot of system have been developed, such as AlxFeCoNiCrTi[2] and AlTiFeNiCuCrx[3], mainly composed of transition elements, which possesses simple crystal structures. Till now, some high entropy alloys has already received a range of generalizations and applications for the cheap costs of development.

\section{The definition and crystal structures}

\subsection{Definition}

According to the theory of Yeh. W. L, high entropy alloys generally consist of more than five main elements. The proportions between principal components are mole ratio or close to the mole ratio. And the contention of main elements is in the range of 5\%-35\%, while the content of minor elements is less than $5 \%$.

\subsection{Crystal Structures}

In the traditional Gibbs phase rule, the number of equilibrium phases equals to $\mathrm{N}+1$ in the system of alloys with $\mathrm{N}$ kinds of main elements. The number of high entropy alloys is not

* Corresponding author: xushuqiong11@,sina.com 
less than $5 \%$. In this concept, high entropy alloys are bound to produce a wide variety of intermetallic compounds, which increased great extent brittleness and reduced the mechanical properties. Fortunately, instead of intricate intermetallic compounds, high entropy alloys formed some simple and common crystal structures such as BCC, FCC, $\mathrm{HCP}$, even the non-crystalline structures [4]. This phenomenon is called high entropy effect [5], which constrained the formation of intermetallic compounds, while forming simple crystal structures.

\section{Properties}

\subsection{High strength and high hardness.}

On the one hand, the alloy will have an intensive lattice distortion effect as a result of uneven atomic radius of various elements. This kind of effect can hinder dislocation movement, which leads to very high strength and hardness. Fig.1. is a schematic diagram of the lattice distortion of high entropy alloy containing Cr. On the other hand, the "cocktail effect"[6] of high entropy alloys intensified the interaction of various alloying elements. Consequently, the high entropy alloy can reflect the comprehensive properties of all kinds of alloy elements. Therefore, the high entropy alloy reflects composite effect improving the strength and hardness.

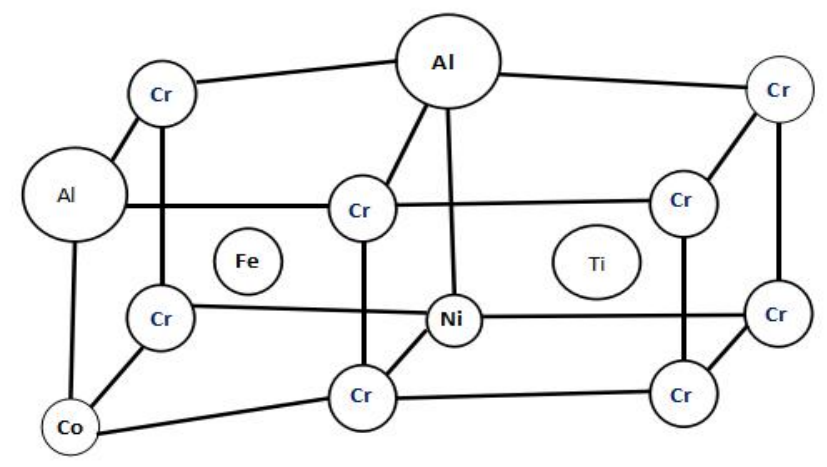

Fig. 1. Schematic diagram of lattice distortion of high entropy alloy containing $\mathrm{Cr}$

As is shown in many experiments, high entropy alloys have higher high hardness. Guo Jianbin[7] melted out of four groups of high entropy alloy, ZrTiHfVNb, ZrTiHfNbMo, ZrTiHfVTa and TaMoWNbZr, and he carried out a compression experiment and measured the values of yield strength, which are respectively $1152 \mathrm{MPa}, 1134 \mathrm{MPa}$, and $1089 \mathrm{MPa}$. However, with the increases in the experimental load, the three kinds of alloys were soon broken, and the measured plastic strain is lower. The value of yield strength of ZrTiHfVNb is only $836 \mathrm{MPa}$, but the alloy showed excellent plasticity and the plastic strain reached $55 \%$.

\subsection{Corrosion resistance}

For one thing, because the crystal structure of high entropy alloy tends to be simplified, the corrosion resistance of high entropy alloys is superior to that of plenty of widely used stainless steel. For another thing, lots of high entropy alloy are dendritic segregation structures[8], which are composed of simple surface structure and body structure. And there are many disordered structures and nanometer particles in dendritic segregation structures. 
The appearance of these structures has greatly increased the corrosion resistance of high entropy alloys. For example, high entropy alloy $\mathrm{Cu} 0.5 \mathrm{NiAlCoCrFeSi}$ at room temperature exhibited better corrosion resistance than 304 and stainless steel in $1 \mathrm{~mol} / \mathrm{L} \mathrm{NaCl}$ and 0 . $5 \mathrm{~mol} / \mathrm{L} \mathrm{H}_{2} \mathrm{SO}_{4}$ solutions[9].

\subsection{Thermal stability}

Entropy is the physical quantity of the system chaos in thermodynamics. The greater entropy means the greater the degree of chaos. According to the function of Gibbs free energy, the entropy of the system (S), temperature $(\mathrm{T})$, enthalpy $(\mathrm{H})$ satisfied the following mathematical relations:

\section{$\mathrm{G}=\mathrm{H}-\mathrm{TS}$}

It can be known that higher entropy value makes the system more stable. And the high temperature can also reduce the free energy of the system, so the high entropy alloys have good thermal stability.

\section{The preparation}

\subsection{Magnetron sputtering.}

Magnetron sputtering[10] is to use certain high energy particle to bombard the surface of a specific material. The process is shown in Fig. 2., argon is placed in a magnetron sputtering device. Under the action of strong electric field, argon wills ionization argon ion and electrons. Then, ions are accelerated toward the cathode in the electric field to bombard target surface with high energy, resulting the occurrence of sputtering target. Therefore, placed in a magnetic field and an electric field with orthogonal distribution, the target material will emit secondary electrons, in which case, the target material will ionize more ions to continue to bombard the target; the target will be sputter deposition to the substrate surface to form a thin film.

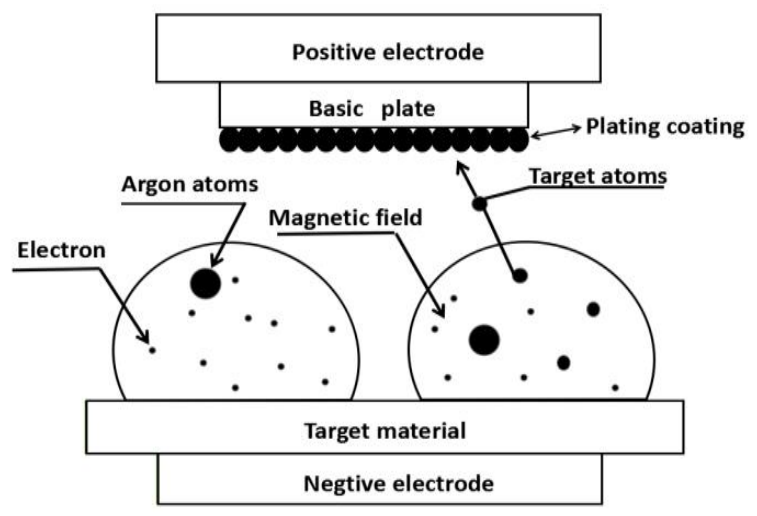

Fig. 2. Magnetron sputtering illustrative diagram

The FeCrCoNiMn high entropy alloy thin films were deposited on 304 stainless steel substrates and Si (100) substrates by magnetron sputtering at Lanzhou University[11]. The results show that the films are uniform and amorphous in a certain sputtering power. But when the sputtering power reaches a certain value, it will get a very rough film and the surface density decreases. With the increasing of sputtering power, the thin films will 
become smaller, and the amorphous structure will also be transformed into crystal structure. Therefore, the sputtering power can directly affect the properties of the film.

\subsection{Vacuum arc melting.}

Here are the brief descriptions of the process of Vacuum arc melting. Firstly, a certain proportion of the pure metal and then placed in the pot, in the vacuum furnace and the air out of the repeated charge of protective gas. Then, the arc discharge is formed between the electrode with the high current and the pot, so that the temperature can be raised so high in a short time, which can make sure the alloy, can be uniformly melted in a short time. And the final step is water cooled casting. The advantages of the method are that the refined alloy has high purity, can prevent pollution, and can refine the metal with high melting point[12].

With Vacuum arc melting, $\mathrm{Cu} 0,5 \mathrm{AlFeNiCrTi}$ and $\mathrm{CuAlFeNiCrTi}$ are melted by Gao Peng[13]. The microstructure, crystal structure, mechanical properties and corrosion resistance of the two alloys were analyzed by a series of experiments. It was found that these two kinds of alloy have a simple crystal structure with excellent mechanical properties and corrosion resistance.

\subsection{Laser cladding}

Laser cladding is a new technology of smelting high entropy alloys in recent years. And it broke through the dimensional limitation of traditional high entropy alloys. And the micro structure of alloys is uniform and compact with fewer micro defects [14].

Qiu Xingwu chose the matrix material of Q235 steel, coated with high entropy alloy $\mathrm{A} 2 \mathrm{CrFeCoCuNixTi}(\mathrm{x}=0,0.5,1,1.5,2)$ with different nickel contents. He found out that the structure of these alloys are simple BCC+FCC through the XRD[15].

\section{Applications}

\subsection{Application of cutting tools.}

Comparing the friction and wear behavior of high entropy coating tool and ordinary high speed steel[16]. When the external load and rotational speed are fixed, the friction of the high entropy alloy coated cutting tool is always lower than that of the ordinary high speed steel. When the tool is rotating at high speed, the higher the friction coefficient is, the higher the friction resistance will be. At this time the chip is very easy to stick knife, and it will scratch the surface of the material, which will reduce the surface finish of the material. Accordingly, the high entropy alloy coating on the surface of the cutting tool can reduce the friction force in the cutting process, which will prolong the tool life and improve the surface quality of the machined surface. Finally, because of high strength and hardness, high entropy alloy coating can be used in high speed cutting.

\subsection{Application of hydrogen storage.}

Many researches and experiments show that some high entropy alloys have excellent hydrogen storage function. Qiu Jianping studied the hydrogen storage properties of these three kinds of high entropy alloys, which are respectively ScTiVCrCo, ScTiVCrNi, $\mathrm{ScTiVCrCu}$. The results show that the maximum hydrogen absorption capacity of the three alloys is respectively $1.76 \%, 2.19 \%$, and $2.56 \%$. With the increase of the number of 
hydrogen absorption, the amount of hydrogen absorption will be reduced correspondingly [17].

\subsection{Application of chemical corrosion.}

Because of eminent corrosion resistance, the high entropy alloy can be applied to all kinds of corrosive working circumstances such as the bottom of ocean ship, the occasions which are prone to electrochemical reaction and various chemical vessels, etc..

\subsection{High load and high impact applications.}

High entropy alloys can be used to produce high quality golf hitting surfaces, high hardness steel pipe and turbine blade, which work in high load and high circumstances. High entropy alloys can also be used for national defense science and technology, such as the track of tanks, artillery shells, etc.

\section{References}

1. X.B.Liang, M.Wei, J.B.Cheng, W.Zhang, Journal of Materials Engineering, 12,7579(2009)

2. W.Li, G.Z.Liu, Rare Metal Materials and Engineering, 29(10),941-944(2009)

3. Y.Liu, M.Chen, Y.X.Li, X.Chen, Acta Metallurgica Sinica, 43(10),1020-1024(2007)

4. C.Z.Yao, H.X.Ma, Chinese Journal of Applied Chemistry, 10, 1189-1194 (2011)

5. Y.Liu, M.Chen, Y.X.Li, Materials Review, 20(4),4-6(2006)

6. C.F.Zhao,Heat treatment, microstructure and properties of some selected multiprincipal elements high-entropy alloys. Fuzhou University(2014)

7. J.B.Guo, Study on microstructure and mechanical properties of five-element refractory high entropy alloys. Beijing Institute of Technology(2015)

8. Z.Y.Sun, Preparation of High Entropy Alloy and Research on Microstructure and Mechanical Properties. Jilin university (2014)

9. J.H.Pi, X.M.Yang, Advanced Materials Industry, 09,71-74(2009)

10. Z.Y.Zheng, Microstructure and Properties of High Entropy Alloy Films Prepared by Direct Current Magnetron Sputtering. Jilin university(2016)

11. Y.Y.Shi, The deposition and the corrosion performance of $\mathrm{Fe} \mathrm{CrCoNiMnCo}$ high entropy alloy thin films. Lanzhou University of Technology(2016)

12. X.N.Yang, W.L.Deng, X.B.Huang, L.H.Tian, Hot Working Technology, 22,3033(2014)

13. P.Gao, H.F.Sun, Z.L.Li, Y.Z.Yan, F.C.Li, H.Y.Zhu, Physical Testing and Chemical Analysis(Physical Testing), 11,677-681(2010)

14. X.Y.Li, H.M.Zhu, Hot Working Technology, 22,30-33(2014)

15. X.W.Qiu, Laser \& Optoelectronics Progress,05,1-12(2017)

16. A.R.Zhang, Li Ye, China Surface Engineering, 04,27-31(2013)

17. J.P.Qiu, Y.C.Luo, Y.L.Liu, K.J.Yang, Hot Working Technology, 02,85-88(2017) 